\title{
Australian specialist palliative care's response to COVID-19: an anonymous online survey of service providers
}

\author{
Tim Luckett ${ }^{1} \wedge$, Andrew Donkor ${ }^{1 \wedge}$, Jane Phillips ${ }^{1} \wedge$, David C. Currow ${ }^{1,2} \wedge$, Deborah Parker ${ }^{1}$, Elizabeth \\ Lobb $^{1,3,4}$, Meera R. Agar ${ }^{1,5}$ \\ ${ }^{1}$ IMPACCT (Improving Palliative, Aged and Chronic Care through Clinical Research and Translation), Faculty of Health, University of Technology \\ Sydney (UTS), Sydney, NSW Australia; ${ }^{2}$ Wolfson Palliative Care Research Centre, University of Hull, Hull, England; ${ }^{3}$ Calvary Health Care, Sydney, \\ Australia; ${ }^{4}$ Faculty of Medicine, University of Notre Dame, Sydney, NSW, Australia; ${ }^{5}$ South Western Sydney Clinical School, University of New South \\ Wales, Sydney, NSW, Australia \\ Contributions: (I) Conception and design: T Luckett, A Donkor, J Phillips, DC Currow, D Parker, MR Agar; (II) Administrative support: A Donkor; \\ (III) Provision of study materials or patients: None; (IV) Collection and assembly of data: T Luckett, A Donkor; (V) Data analysis and interpretation: \\ T Luckett, A Donkor; (VI) Manuscript writing: All authors; (VII) Final approval of manuscript: All authors. \\ Correspondence to: Dr. Tim Luckett. IMPACCT (Improving Palliative, Aged and Chronic Care through Clinical Research and Translation), Faculty of \\ Health, University of Technology Sydney, Building 10, Ultimo, NSW 2007 Australia. Email: Tim.Luckett@uts.edu.au.
}

\begin{abstract}
Background: The corona virus disease 2019 (COVID-19) pandemic has required specialist palliative care (SPC) services to respond by: (I) integrating infection prevention/control measures into care for their usual caseloads and (II) providing consultations and/or care for people dying from a new disease entity. The aim of the current study was to learn about the response of Australian SPC services to COVID-19 and its consequences in order to inform pandemic practice and policy.

Methods: A cross-sectional, anonymous survey was administered online from May to July 2020. Email invitations were sent to 160 providers delivering 503 SPC services listed in the Australian Palliative Care Services Directory. Survey questions asked about service responses to COVID-19, impacts on care quality, and perceived benefits/disadvantages for palliative care clients post-pandemic. Open-ended responses were thematically coded using an established framework that classifies SPC pandemic responses under: 'stuff', 'staff', 'space', 'systems', 'separation', 'sedation', 'communication' and 'equity'.

Results: Complete survey responses were received from 28 providers on behalf of 100 SPC services (response rates of 17\%/20\% respectively): 29 consultative, 25 community home-based, 21 outpatient, 15 inpatient wards/units, eight inpatient hospice and two other services. Responses were reported across all framework categories except 'sedation'. Concerns centred on: inadequate support for self-management, psychosocial needs and bereavement for clients living at home; pressures on staff capacity and wellbeing; and a perceived lack of health system preparedness for a potential future surge. Rapid implementation of telehealth across Australia was perceived to offer potential benefits to palliative care in the longer term, if provided with ongoing support.
\end{abstract}

Conclusions: Meeting COVID-19-related challenges requires SPC to be agile and responsive. Advocacy is required to ensure the needs of people dying and their families are supported as well as people requiring acute care for COVID-19. Expansion of telehealth during the pandemic presents an opportunity for leveraging to benefit palliative care longer term.

Keywords: Corona virus disease 2019 (COVID-19); specialist palliative care (SPC); survey

Submitted Sep 01, 2020. Accepted for publication Nov 23, 2020.

doi: 10.21037/apm-20-1760

View this article at: http://dx.doi.org/10.21037/apm-20-1760

\footnotetext{
^ ORCID: Tim Luckett, 0000-0001-6121-5409; Andrew Donkor, 0000-0002-6073-524X; Jane Phillips, 0000-0002-3691-8230; David C. Currow, 0000-0003-1988-1250; Meera R. Agar, 0000-0002-6756-6119.
} 


\section{Introduction}

The corona virus disease 2019 (COVID-19) pandemic has challenged health systems and services around the world (1). Guidance statements for specialist palliative care (SPC) have been produced by international and national organisations $(2-7)$ and independent authors (8-11). Such guidance is aimed at ensuring safe and high quality care both to SPC's usual caseload and a new caseload of people dying from COVID-19. Recommendations have focused on ensuring: workforce capacity (including operational surge needs); medication, equipment and supplies; infection prevention and control; management of COVID-19 symptoms; and routine advance care planning (ACP).

To date, four published studies have described the responses of SPC services to COVID-19 internationally. A survey of 16 Italian hospices found responses included: redeploying of clinical staff from inpatient to community settings; implementing a telephone triage system; reviewing visiting and care-after-death policies; and providing written guidance on managing confirmed or suspected COVID-19 cases (12). In the UK and Ireland, a survey of 261 clinicians across settings found the COVID-19 pandemic had increased anticipatory prescribing for end-of-life care and changes in route of administration to withstand staff shortages (13). In Taiwan, a survey of 76 hospice inpatient wards found that nearly all had changed their visiting policies, and some had implemented checks of visitor identities and travel histories (14). A longitudinal audit of 19,900 clients' records discharged from Taipei City Hospital found the utilisation of hospice inpatient care services to have reduced during the advent of COVID-19 (15).

No research has yet focused on the response to COVID-19 among Australian SPC services. At the time of the current study, Australia had lower rates of infection and death from COVID-19 than most other countries, with only 335 cases and 4 deaths per million people by the end of the project period (7th July 2020) (16). While rates of COVID-19 infection and death remained low, health authorities in most Australian states and territories nonetheless required strict infection prevention and control procedures, including spatial distancing and use of personal protective equipment (PPE). Non-essential surgery had also been cancelled to free-up hospitals in the event of a surge.

The aim of the current study was to learn about the response of Australian SPC services to COVID-19 and its consequences in order to inform pandemic practice and policy.
We present the following article in accordance with the STROBE reporting checklist (available at http://dx.doi. org/10.21037/apm-20-1760) (17).

\section{Methods}

\section{Design}

A cross-sectional survey study was conducted from May to July 2020. A survey study was used in preference to a qualitative approach to enable sampling of a large number of services in a timely enough way for findings to inform ongoing responses to COVID-19 from within the sector. The survey was administered online using the SurveyMonkey ${ }^{\circledR}$ platform (SurveyMonkey ${ }^{\circledR}$, Paulo Alto, CA, USA). Survey responses were anonymous to reduce social desirability bias and promote honest reporting. To maintain anonymity, completion of the survey was taken as sufficient evidence of consent to participate without an additional informed consent procedure. The study was considered nil/negligible risk in accordance with human research ethics policy at the University of Technology Sydney, Australia. The study was conducted in accordance with the Declaration of Helsinki (as revised in 2013).

\section{Participants and recruitment}

Respondents were eligible if they self-identified as being involved in planning the response to COVID-19 of one or more SPC services. SPC was defined in accordance with the 2018 Australian Palliative Care Service Development Guidelines as "care services comprising multidisciplinary teams with specialised skills, competencies, experience and training in palliative care" (p.7), regardless of the setting in which such care might be delivered (18).

Respondents were recruited using email invitations sent to all 160 SPC service providers listed nationally in the Australian Palliative Care Services Directory (19). According to the Directory, these providers delivered 503 services across the following 'service types': consultative (including acute, subacute and long-term care), outpatient, inpatient wards/units, inpatient hospice and community home-based. A reminder email was sent to all service providers after two weeks.

\section{Data collection}

In the absence of any validated precedent, the survey 
Table 1 A palliative care pandemic framework, adapted from Downar and Seccareccia [2010] (10), Etkind et al. [2020] (11) and Arya et al. [2020] (8)

Stuff: medical equipment, medication, personal protective equipment

Staff: workforce capacity (including numbers and expertise)

Space: hospital wards and other places of care and work for healthcare professionals

Systems: policies, systems and processes for delivering care

Separation: hospital and long-term care visitor restrictions

Sedation: capacity to sedate clients with refractory symptoms

Communication: advance care planning.

Equity: ways of ensuring the most vulnerable and marginalised clients receive care

questions were developed ad hoc with input from palliative care physicians (DCC, MRA), nurses (JP, DP) and bereavement counsellor (EL) on the authorship team, who included people with SPC service management experience (DCC, MRA). Closed questions asked about service number, type(s) and location [state/territory and remoteness (20)]. Open-ended questions asked about service changes that were planned or had occurred in response to COVID-19 in relation to the six domains of care defined by the Australian National Palliative Care Standards (21), namely: assessment of needs; developing the care plan; caring for carers; providing care; transitions within and between services; and grief support. Respondents were asked to indicate any additional resources required to implement changes, impacts on service quality and outcomes, and any lessons learned. Further questions asked about perceived benefits and disadvantages of service changes to future care of SPC's usual caseload beyond the pandemic, and SPC's preparedness and challenges in caring for clients dying from COVID-19. Open-ended questions were used in preference to closed questions to enable respondents to choose which service responses to the pandemic were most important to focus on and so capture a diversity of experience with feasible response burden. Respondents were also asked to provide brief information about themselves, including their discipline, involvement in COVID-19-related service planning, and any new responsibilities they had taken on as a result.

\section{Analysis}

Data were imported into NVivo v12 (QSR International) for management. Analysis of responses to open questions followed a process of familiarisation and line-by-line open coding against a framework that has been used by several authors to classify SPC's response to pandemics, and groups responses under categories of 'stuff', 'staff', 'space', 'systems', 'separation', 'sedation', 'communication' and 'equity' (see Table 1) $(8,10,11)$. Codes were added inductively to accommodate any responses not well-described by previous versions of the framework. Analysis was conducted by one researcher $(\mathrm{AD})$, with review and discussion with a second (TL) after the first three survey responses to agree a coding framework, and at regular intervals thereafter. It was not deemed necessary to double code each response because they tended to be brief, explicit and convey only one idea.

\section{Statistical analysis}

Responses on quantitative items were analysed using descriptive statistics, using SPSS Statistics V25 (IBM).

\section{Results}

Twenty-eight respondents completed sufficient survey questions to be included in the analysis, representing $17 \%$ of the SPC providers listed on the PCA Directory $(n=160)$. Of these, 22 were nurses and nine doctors, with three respondents identifying both disciplines and one also identifying an administrator role. Seventeen respondents acknowledged taking on new responsibilities as a result of COVID-19.

Respondents reported on behalf of 100 services (20\% of SPC services listed in the Directory), with a median of 2 services each (inter-quartile range, 1-3.25). Eleven respondents reported on behalf of services in major cities, 12 inner regional, 12 outer regional, and 6 remote. The numbers of services of each type in each Australian state and territory are summarised in Table 2 .

Participants took a median of 21 minutes to complete the survey (inter-quartile range, 12-60 minutes). 
Table 2 Numbers of Australian specialist palliative care services represented from each state $(\mathrm{N}=100)$

\begin{tabular}{|c|c|c|c|c|c|c|}
\hline Service type & \multicolumn{6}{|c|}{ State } \\
\hline Consultative & 12 & 11 & 3 & 2 & 1 & 29 \\
\hline Community home-based & 6 & 9 & 6 & 3 & 1 & 25 \\
\hline Outpatient & 3 & 6 & 9 & 3 & 0 & 21 \\
\hline Inpatient hospice & 1 & 1 & 3 & 2 & 1 & 8 \\
\hline Other* & 0 & 0 & 0 & 1 & 1 & 2 \\
\hline Jurisdictional totals & 29 & 31 & 23 & 13 & 4 & \\
\hline
\end{tabular}

${ }^{*}$ Other, a service for prisons, and telehealth support for neighbouring health districts. Vic, Victoria; NSW, New South Wales; SA, South Australia; QId, Queensland; WA, Western Australia.

\section{Service changes, resource requirements and impact on care quality}

Respondents reported changes in all of the palliative care framework domains except for sedation. An additional code was added under 'communication' to accommodate changes in communication with clients not previously mentioned in the literature.

Several respondents highlighted the need for SPC services to remain agile and responsive within the context of changing and unpredictable COVID-19 case numbers. Innovation and collaboration with other service providers were reported to be further factors for determining success. Several respondents highlighted the increase in time required to plan for and deliver care during the COVID-19 pandemic.

See Table 3 for a summary of changes reported to have been implemented or planned. There were no discernible patterns with regard to jurisdiction or remoteness. Issues specific to particular service types are outlined below.

\section{Stuff}

Equipment needs highlighted by respondents included PPE, COVID-19 testing kits, and additional medicines and equipment (syringe drivers, oxygen concentrators). Several respondents identified a difficulty in obtaining sufficient PPE, especially at the start of the pandemic. For one community service, this meant a reluctance to accept referrals for COVID-19-positive clients discharged from hospital. Stockpiling of medications included 'grab and go' subcutaneous medication packs for long-term care.

\section{Staff}

Changes to-and pressures on-staffing were among the most commonly reported consequences of COVID-19. Increased demands were placed on staffing by the need to prevent/control infection and undertake new duties conferred by the pandemic.

Provision of staff education on the proper use of PPE was reported to be a key infection prevention/control measure. However, respondents often expressed barriers to PPE compliance, including its perceived interference with assessment and communication of empathy, and the additional time required to use it. One respondent reporting on behalf of outer regional and remote community-based services in Victoria also reported that PPE and hand sanitiser had occasionally been stolen from staff vehicles.

In addition to PPE, reported measures for infection prevention/control included: splitting staff into separate teams and minimising face-to-face meetings; allocating older and/or unwell staff members to tasks that enabled them to work from home; ceasing volunteer activities; and minimising the number of home visits and staff attending these. New duties required by COVID-19 were reported to include screening clients and visitors to hospital services, and additional education, training and support for clients in the community. Several respondents acknowledged the fragility of staff capacity and service delivery in the event of an infection.

"If one member [gets] sick, [we] could very easily have [the] entire department in self-isolation" [Respondent (R)4, reporting on behalf of inner and outer regional and remote consultative services in Victoria]. 
Table 3 Changes to Australian specialist palliative care services implemented or planned in response to the COVID-19 pandemic

\begin{tabular}{|c|c|c|}
\hline Domains & SPC response & Change \\
\hline \multirow{2}{*}{ Stuff } & \multirow{2}{*}{ Ensure supply } & Coordinate and collaborate across jurisdictions to expedite access \\
\hline & & $\begin{array}{l}\text { Emergency medicines (e.g., 'grab and go' subcutaneous medication packs for long-term } \\
\text { care) }\end{array}$ \\
\hline \multirow{3}{*}{ Staff } & \multirow{2}{*}{ Protect capacity } & Cease volunteer activities and redeploy vulnerable staff to lower risk work \\
\hline & & Reduce home visits, number of staff attending these, and consultation time \\
\hline & Build capacity & Create new roles (e.g., inpatient visitor screening) \\
\hline Space & Infection prevention/control & Rearrange office space to enable spatial distancing \\
\hline \multirow[t]{5}{*}{ Systems } & \multirow{2}{*}{$\begin{array}{l}\text { Amend existing triage } \\
\text { system }\end{array}$} & Add COVID-19 risk rating, screening and testing \\
\hline & & Add telephone triage for face-to-face contact versus telehealth \\
\hline & \multirow[t]{2}{*}{ Use of telehealth } & Train and provide ongoing IT support for staff and clients \\
\hline & & Supply equipment (e.g., tablets) to clients \\
\hline & Local guidance & Develop protocols for caring for COVID-19 positive clients \\
\hline \multirow{2}{*}{ Separation } & \multirow{2}{*}{ Visitor policies for inpatients } & $\begin{array}{l}\text { Visitors temperature checked and asked to complete survey indicating symptoms, travel } \\
\text { and contacts }\end{array}$ \\
\hline & & Creation of separate COVID-19 wards \\
\hline \multirow[t]{6}{*}{ Communication } & \multirow[t]{4}{*}{ Support clients } & Provide correct, constant and consistent information on COVID-19 response \\
\hline & & Appoint designated staff to address client information needs \\
\hline & & Send daily email updates on each inpatient to families \\
\hline & & Provide clear signs on change in policies at facility entrances \\
\hline & \multirow[t]{2}{*}{ ACP } & Increase uptake of ACP \\
\hline & & Update existing ACPs with considerations relating to COVID-19 \\
\hline Equity & Streamline access & Remove avoidable barriers to telehealth, particularly in remote areas \\
\hline
\end{tabular}

ACP, advance care planning; PPE, personal protective equipment; SPC, specialist palliative care; IT, information technology. 
Three respondents reported that inpatient restrictions on visitors had led to increased conflict between clients and staff, with one mentioning violence. Respondents recommended strategies such as debriefing, formal systems enabling peer-to-peer support and self-care, and close contact with appropriate mechanisms to channel daily updates on COVID-19.

Many respondents also reported an increase in the need for staff to provide psychosocial and spiritual support for clients, families and carers, which was exacerbated by the withdrawal of some dedicated counselling services. Concern was expressed by one respondent that there might be an increase in complicated grief among family members of people dying during the pandemic. Another respondent indicated that their service planned to hold a memorial service after the COVID-19 pandemic to acknowledge everyone who had died during this period.

However, these challenges were qualified by significant strengths perceived to characterise SPC's response to COVID-19, especially staff's expertise and experience in $\mathrm{ACP}$ and providing support to people anxious about dying.

\section{Space}

Respondents indicated that, during the initial stages of the pandemic, service managers had anticipated that facilities might be overwhelmed by demand on extra beds to care for large numbers of clients dying of COVID-19. While this crisis had not transpired, respondents reported that inpatient services were continuing to keep wards allocated for COVID-19 positive clients to control transmission of infection. Other space-related strategies mentioned by respondents included a long-term care facility which was perceived to be exemplary for having single fully selfcontained rooms with external doors to enable visitors while preventing infection to other residents. Another respondent reported that SPC office space had been rearranged to increase distancing between staff, as well as staff being encouraged to work from home where possible.

\section{Systems}

Respondents reported leveraging existing systems to: assess initial and ongoing needs of clients; plan, communicate and support decisions about care; ensure seamless transitions within and between services; and provide bereavement and grief support services. Nearly all respondents reported maximising the use of telehealth to reduce face-to-face contact. Telehealth was reportedly used for almost all aspects of care, including needs assessment, developing a care plan, providing education and support for clients, delivering care to clients, supporting transfer of clients between and within services, and providing bereavement support to families following a client death. Respondents reported greater efficiencies from telehealth over face-toface contact in the form of reduced travel time, leading to increased access to services in regional and remote areas and improved follow-up more generally. However, views were mixed on the quality of care delivered via telehealth versus face-to-face, with some perceiving it to be inferior for assessment, carer training and psychosocial support, but others claiming there to be little difference. There were also mixed reports regarding acceptance of telehealth among staff and clients, with one respondent reporting this to be better than expected but others identifying attitudinal barriers to uptake. Respondents also identified several technical barriers to telehealth, including: time demands for set-up; high cost of telehealth equipment; limited access to high speed internet in remote areas; and system malfunction.

"One of the biggest challenges relating to undertaking preparedness for this team, was identifying and setting up the required ICT systems and devices to support this initiative [telehealth], some of this is still a work in progress" (R11, reporting on behalf of outer regional and remote consultative, outpatient and community home-based services in New South Wales).

Several respondents referred to the rapid development and dissemination of care guidelines and standards, including those for caring for COVID-19 positive clients, infection prevention/control, and bereavement support. However, one respondent reporting for an outer regional community home-based service expressed concerns about the lack of guidelines for caring for COVID-19 positive clients in the community.

Existing triage systems were amended to include assessment of need for face-to-face contact versus telehealth and COVID-19 risk rating, screening and testing prior to contact.

Several respondents reported a decline in referrals and admissions to palliative care inpatient units, which they linked to cancellation of elective surgeries and families wanting to avoid admissions due to restricted hospital visiting hours. 


\section{Separation}

Inpatient service providers nearly all reported changes in visitor policies leading to a reduction in visiting hours and/ or number of visitors. Respondents commonly reported commensurate concerns regarding increased loneliness, social isolation and anxiety in clients, families and carers. However, one respondent indicated that visitor restrictions were sometimes relaxed at the end-of-life to enable family members to say goodbye.

Respondents emphasised the importance of maintaining social connection between clients and family members by supplying them with tablets to enable videoconferencing, which was reportedly preferred by many clients compared to telephone.

\section{Communication}

Respondents expressed concern over what they described to be a decline in communication regarding care plans during client transfer between settings. An increased need for reliable, frequent and consistent communication of information was highlighted by several respondents as being a core component to ensuring optimal care continuity and coordination. One respondent reported setting up an email service to send daily updates on inpatient progress to designated family members who then forwarded these to others.

\section{Equity}

Comments on equity were largely concerned with tensions between the dual potentials for telehealth to enhance access on the one hand while leading to a digital divide on the other. To optimise equitable benefits from telehealth, respondents highlighted the need to support vulnerable clients with education and training, provision of tablets and other equipment, and IT technical support.

\section{Perceived longer-term benefits and disadvantages of COVID-19-related changes to care of SPC's usual caseload}

The rapid expansion of telehealth during the pandemic was widely celebrated as offering potential to confer ongoing benefits in terms of timely access for clients and efficiencies for services. However, there was also a concern that such efficiencies might lead to telehealth being overextended beyond the purposes for which it was best suited, reducing quality of care where face-to-face contact was considered optimal. Respondents also expressed a hope that initiatives supporting staff welfare and team communication during the pandemic would be continued into the future.

Finally, several respondents identified more general shifts that they hoped would be sustained into the postCOVID-19 future. These included perceived increases in societal acceptance of ACP, and services' willingness to review procedures and engage in problem solving.

"A willingness to work with ambiguity and to provide flexible planning with limited resources and information”. (R6, reporting on behalf of inner and outer regional consultative, outpatient and community home-based services in New South Wales).

\section{SPC's preparedness to care for people dying with COVID-19}

Eight respondents indicated that a service on behalf of which they reported had been involved in providing care to clients with COVID-19, four of whom reported delivering care directly or in a consultative capacity to a single client, and one of whom reported developing a model of care for COVID-19 positive clients without specifying whether this had been used.

Most respondents were confident in the expertise SPC could provide to facilitate decisions about whether or not to pursue life-sustaining treatments, alleviate COVID-19related symptoms at the end-of-life, and support family members through end-of-life and into bereavement. However, there was concern that the quality of such care might be compromised by infection prevention/control procedures, and that families of community-based cases might be at high risk of infection.

Two respondents reported a belief that the healthcare response as a whole had overlooked the needs of clients dying from COVID-19 and their families, with an emphasis on 'save and rescue' rather than palliation and SPC underinvolved in planning, resourced and utilised. These and several other respondents perceived there to be suboptimal capacity both within SPC and the COVID-19 response more generally to cope with a potential surge in cases, especially within long-term care.

"If there is a surge as has been seen in other countries we simply don't have the capability to care for the numbers of potential patients". (R9, reporting on behalf of inner regional inpatient ward/unit, consultative, outpatient and community home-based services in Victoria). 


\section{Discussion}

This study has provided insights into the changes implemented and planned by Australian SPC services in response to the COVID-19 pandemic up to mid-2020. SPC services were reported to have made significant changes to support infection prevention and control, including changes to visitor policies for inpatient services, limiting home visits for community-based services, and use of PPE and physical distancing across all service types as directed by public health orders. While access to telehealth and other technologies were perceived to have ameliorated potential impacts on service access, concerns remained that some clients were under-supported in the community with regard to self-management, psychosocial and bereavement needs. There was also widespread concern regarding staff capacity and wellbeing. As we anticipated from the small number of deaths from COVID-19 in Australia up until the time of data collection, services reported limited experience of caring for people dying with COVID-19. Respondents reported confidence in their expertise to manage COVID19-related symptoms and family distress, but some felt under-resourced and under-involved in planning for the health system to be adequately prepared for a potential escalation of deaths in the future. Respondents emphasised the need for services to be agile and responsive in the pandemic's rapidly-changing context, as has been highlighted by other authors (9).

The need for greater preparation of the Australian response has also been emphasised by the Australian Coronavirus Disease 2019 (COVID-19) Palliative Care Working Group (ACPCWG), a collaboration of national organizations initiated by Australia's peak body for palliative care, Palliative Care Australia (22-24). The ACPCWG has highlighted the need to model operational surge capacity to identify how SPC might best support other services in delivering palliative care (e.g., emergency departments, intensive care, respiratory care and long-term care) in the event that a large number of dying patients overwhelms SPC's capacity to care for everyone directly. The ACPCWG has also advocated for: inclusion of palliative medicines in the Therapeutic Goods Administration's (TGA) Medicines Watch List (25) to ensure national supplies; transparency regarding triage guidelines and the ethical principles underpinning these in the event healthcare resources are rationed; consistency among visitors policies regarding compassionate grounds; and planning for bereavement care post-pandemic. The ACPCWG through CareSearch has established a centralised web portal (26) for accurate, up-to-date and evidence-based information on palliative care during the pandemic, as well as contributed to the National COVID-19 Clinical Evidence Taskforce (27) to ensure implications for palliative care and older people are considered in more general evidence-based recommendations to Australia's healthcare community. At the same time, however, it is noteworthy that the Australian Health Sector Emergency Response Plan for COVID-19 published by the Department of Health makes no mention of palliative care or SPC (28).

As well as highlighting the need for SPC to be involved in national and local epidemic/pandemic planning, published advice on palliative care during pandemics has suggested that collaborations between services be formed to give mutual support and coverage if staff become sick or overwhelmed, and that standardised information be collected across services to enable continuous monitoring/ evaluation $(8,11)$. Perceptions regarding a lack of preparedness reported in our study echo findings from an earlier Italian survey of SPC hospices (12); however, unlike Italy in early 2020, Australia has the considerable advantage of hindsight regarding other countries' mistakes.

The COVID-19 pandemic has created a worldwide imperative to implement telehealth (29-31). The Australian response has been second to none in extending free-touser access to telehealth, removing previous limits on remoteness and clinical context (32). Continuing telehealth to palliative care clients post-pandemic will enable access to specialist services not only for clients in regional and remote areas but also for clients in major cities who may be too unwell to travel but were ineligible for telehealth prior to COVID-19. However, respondents in our study varied regarding their degree of satisfaction with telehealth and views on its suitability for the different domains of palliative care. Research has found mixed evidence for benefits from telehealth for client outcomes in palliative care $(33,34)$, and its full potential requires a range of supports including technical assistance with system set-up, skills development, ongoing investment in equipment, technical support, and infrastructure investment to enable high-speed internet access in remote areas (35). Further research is needed to leverage the learnings forced on SPC by COVID-19 to inform optimal telehealth post-pandemic (36).

Respondents to the current survey expressed particular concern regarding bereavement outcomes of family members of people dying during the pandemic. Infection 
prevention and control measures have disrupted families' usual experiences of bereavement by limiting physical access to dying loved ones, reducing opportunities to collectively mourn (e.g., at funerals) and receive ongoing support from professional services and social networks (37). Deaths during COVID-19 have occurred within a milieu of other losses relating to routine, freedom, trust in others and future plans (38). These secondary losses are likely to complicate normal grief reactions by compounding distress and removing meaningful activities that may provide a respite. Greater attention to bereavement care may therefore be needed to prevent prolonged grief disorder becoming a major mental health issue in the postCOVID-19 world (39).

\section{Strengths and limitations}

The generalisability of findings from the current research are limited by the low response rate and inevitable volunteer effect associated with survey studies of this kind (40). More than $80 \%$ of respondents and services were drawn from three states (Victoria, New South Wales or South Australia), and there was no representation from Australia's three least-populous jurisdictions (Australian Capital Territory, Northern Territory and Tasmania). While further bias cannot be characterised with certainty, it seems likely that participating services may have been more proactive than average in responding to the COVID-19 pandemic, or else that respondents were more concerned than average about its impacts on quality of care. Nonetheless, our findings were highly consistent with those of previous survey studies from other countries (12-15) and international guidance (2-6,8-11).

The anonymous nature of the survey reduced the risk of social desirability bias. However, it also made it impossible to rule out the possibility that some services were reported by more than one respondent, despite email invitations being sent to only one address and stressing the need for service-level reporting. Also, the service types, numbers and providers targeted for recruitment were themselves subject to inaccuracies in the Australian Palliative Care Services Directory (19), with several contact details found to be erroneous. Finally, findings were limited by the brief survey responses given by most respondents, and future research using focus groups and/or semi-structured interviews is recommended to provide a more in-depth exploration of issues highlighted as salient, such as preparedness to care for people dying from COVID-19.

\section{Conclusions}

Meeting COVID-19-related challenges requires SPC to be agile and responsive and more connected to the health system in general. Advocacy is required to ensure the needs of people dying and their families are supported as well as people requiring acute care for COVID-19. Rapid expansion of telehealth during the pandemic represents its greatest opportunity for palliative care longer-term, provided that appropriate support is ongoing and quality as well as efficiency drives decisions about where it should - versus should not-replace face-to-face care. The scale and intensity of telehealth uptake should be matched by commensurate attention from research to leverage learnings for optimising care delivery and outcomes post-pandemic.

\section{Acknowledgments}

We would like to acknowledge the involvement of Ingrid Amgarth Duff who worked as an IMPACCT research assistant on approaching SPC services for this study.

Funding: Funding for this study was from discretionary funds held by IMPACCT, Faculty of Health, University of Technology Sydney.

\section{Footnote}

Reporting Checklist: The authors have completed the STROBE reporting checklist. Available at http://dx.doi. org/10.21037/apm-20-1760

Data Sharing Statement: Available at http://dx.doi. org/10.21037/apm-20-1760

Conflicts of Interest: All authors have completed the ICMJE uniform disclosure form (available at http://dx.doi. org/10.21037/apm-20-1760). MRA and JP are members of the Australian Coronavirus Disease 2019 (COVID-19) Palliative Care Working Group (ACPCWG) referred to in the discussion of this manuscript. The other authors have no conflicts of interest to declare.

Ethical Statement: The authors are accountable for all aspects of the work in ensuring that questions related to the accuracy or integrity of any part of the work are appropriately investigated and resolved. The study was considered low/negligible risk according to the human research ethics policy at the University of Technology 
Sydney (ETH20-4917), and informed consent was taken as implied by completion of the online survey to maintain anonymity. The study was conducted in accordance with the Declaration of Helsinki (as revised in 2013).

Open Access Statement: This is an Open Access article distributed in accordance with the Creative Commons Attribution-NonCommercial-NoDerivs 4.0 International License (CC BY-NC-ND 4.0), which permits the noncommercial replication and distribution of the article with the strict proviso that no changes or edits are made and the original work is properly cited (including links to both the formal publication through the relevant DOI and the license). See: https://creativecommons.org/licenses/by-nc-nd/4.0/.

\section{References}

1. Lacina L. COVID-19 reveals gaps in health systems: WHO briefing. Geneva: World Economic Forum; 2020.

2. Gilissen J, Pivodic L, Unroe KT, et al. International COVID-19 Palliative Care Guidance for Nursing Homes Leaves Key Themes Unaddressed. J Pain Symptom Manage. 2020. Available online: https://doi.org/10.1016/ j.jpainsymman.2020.04.151

3. Worldwide Palliative Care Alliance. COVID-19: end of life care at home: guidance for family members and friends. London: WPCA; 2020.

4. Association for Palliative Medicine of Great Britain and Ireland. COVID-19 and palliative, end of life and bereavement care in secondary care role of the specialty and guidance to aid care. APM; 2020:1-39.

5. Ontario Palliative Care Network. Planning for palliative care delivery during the COVID-19 pandemic. Ontario: Ontario Palliative Care Network; 2020.

6. Bear L, Simpson N, Angland M, et al. 'A good death'during the Covid-19 pandemic in the UK: a report on key findings and recommendations. London: London School of Economics and Political Science; 2020:1-18.

7. Australian COVID-19 Palliative Care Working Group. COVID-19 updates 2020 Available online: https:// palliativecare.org.au/covid-19-updates

8. Arya A, Buchman S, Gagnon B, et al. Pandemic palliative care: beyond ventilators and saving lives. CMAJ 2020;192:E400.

9. Pahuja M, Wojcikewych D. Systems barriers to assessment and treatment of COVID-19 positive patients at the end of life. J Palliat Med 2021;24:302-4.

10. Downar J, Seccareccia D. Palliating a pandemic: "all patients must be cared for". J Pain Symptom Manage 2010;39:291-5.

11. Etkind SN, Bone AE, Lovell N, et al. The role and response of palliative care and hospice services in epidemics and pandemics: A rapid review to inform practice during the COVID-19 pandemic. J Pain Symptom Manage 2020;60:e31-e40.

12. Costantini M, Sleeman KE, Peruselli C, et al. Response and role of palliative care during the COVID-19 pandemic: a national telephone survey of hospices in Italy. Palliat Med 2020;34:889-95.

13. Antunes B, Bowers B, Winterburn I, et al. Anticipatory prescribing in community end-of-life care in the UK and Ireland during the COVID-19 pandemic: online survey. BMJ Support Palliat Care 2020;10:343-9.

14. Hsu YC, Liu YA, Lin MH, et al. Visiting Policies of Hospice Wards during the COVID-19 Pandemic: An Environmental Scan in Taiwan. Int J Environ Res Public Health 2020;17:2857.

15. Chou YC, Yen YF, Feng RC, et al. Impact of the COVID-19 Pandemic on the Utilization of Hospice Care Services: A Cohort Study in Taiwan. J Pain Symptom Manage 2020;60:e1-e6.

16. Australian Government Department of Health. Australian Government Department of Health Coronavirus (COVID-19) Current Situation and Case Numbers Canberra; 2020.

17. von Elm E, Altman DG, Egger M, et al. Strengthening the Reporting of Observational Studies in Epidemiology (STROBE) statement: guidelines for reporting observational studies. BMJ 2007;335:806-8.

18. Palliative Care Australia. Palliative Care Service Development Guidelines. Melbourne; 2018.

19. Palliative Care Australia. National palliative care services directory Canberra: Palliative Care Australia; 2020. Available online: https://palliativecare.org.au/directory-ofservices

20. Australian Bureau of Statistics. Australian Statistical Geography Standard (ASGS): Volume 5 - Remoteness Structure. Canberra: ABS; 2016. Contract No.: 1270.0.55.005.

21. Palliative Care Australia. National palliative care standards. 5 ed. Canberra: Palliative Care Australia; 2018.

22. Cairns W, Agar M. Integrating palliative care into COVID-19 planning. Insight + [Internet]. 2020;11: [23 March 2020]. Available online: https://insightplus.mja. com.au/2020/11/integrating-palliative-care-into-covid-19planning/ 
23. Agar M, on behalf of the Australian COVID-19 Palliative Care Working Group. Australian COVID-19 Palliative Care Working Group COVID-19: Why palliative care matters! Palliative Care Australia [Internet]. 2020: [2nd April 2020]. Available online: https://palliativecare.org.au/ wp-content/uploads/2020/04/COVID-19-PC-WorkingGroup-Why-palliative-care-matters_02042020.pdf

24. Palliative Care Australia. Statement on Coronavirus Disease 2019 (COVID-19) 2020: [March 2020]. Available online: https://palliativecare.org.au/wp-content/uploads/ dlm_uploads/2020/03/Palliative-Care-Australiastatement-FINALupdated-2603.pdf

25. Australian Department of Health Therapeutic Goods Administration. Medicine shortages guidance and resources 2020. Available online: https://www.tga.gov.au/ medicine-shortages-guidance-and-resources

26. Care Search. COVID-19 2020. Available online: https:// www.caresearch.com.au/caresearch/tabid/5950/Default. aspx

27. National COVID-19 Clinical Evidence Taskforce. Caring for people with COVID-19; 2020. Available online: https://covid19evidence.net.au/

28. Australian Commonwealth Department of Health. Australian health sector emergency response plan for novel Coronavirus (COVID-19). Canberra: Dep't Health; 2020.

29. DiGiovanni G, Mousaw K, Lloyd T, et al. Development of a telehealth geriatric assessment model in response to the COVID-19 pandemic. J Geriatr Oncol 2020;11:761-3.

30. Calton B, Abedini N, Fratkin M. Telemedicine in the time of coronavirus. J Pain Symptom Manage 2020;60:e12-e14.
31. Humphreys J, Schoenherr L, Elia G, et al. Rapid implementation of inpatient telepalliative medicine consultations during COVID-19 pandemic. J Pain Symptom Manage 2020;60:e54-e59.

32. Fisk M, Livingstone A, Pit SW. Telehealth in the Context of COVID-19: Changing Perspectives in Australia, the United Kingdom, and the United States. J Med Internet Res 2020;22:e19264.

33. Head BA, Schapmire TJ, Zheng Y. Telehealth in palliative care: a systematic review of patient-reported outcomes. Journal of Hospice \& Palliative Nursing 2017;19:130-9.

34. Zheng Y, Head BA, Schapmire TJ. A systematic review of Telehealth in palliative care: caregiver outcomes. Telemed J E Health 2016;22:288-94.

35. Australian Digital Health Agency (ADHA). Australia's National Digital Health Strategy. Canberra: ADHA; 2018.

36. Chua IS, Jackson V, Kamdar M. Webside Manner during the COVID-19 Pandemic: Maintaining Human Connection during Virtual Visits. J Palliat Med 2020;23:1507-9.

37. Wallace CL, Wladkowski SP, Gibson A, et al. Grief during the COVID-19 pandemic: considerations for palliative care providers. J Pain Symptom Manage 2020;60:e70-e76.

38. Breen LJ. Grief loss and the COVID-19 pandemic. Aust J Gen Pract 2020. doi: 10.31128/AJGP-COVID-20.

39. Eisma MC, Boelen PA, Lenferink LIM. Prolonged grief disorder following the Coronavirus (COVID-19) pandemic. Psychiatry Res 2020;288:113031.

40. Eysenbach G, Wyatt J. Using the Internet for Surveys and Health Research. J Med Internet Res 2002;4:E13.
Cite this article as: Luckett $\mathrm{T}$, Donkor A, Phillips J, Currow DC, Parker D, Lobb E, Agar MR. Australian specialist palliative care's response to COVID-19: an anonymous online survey of service providers. Ann Palliat Med 2021;10(3):2747-2757. doi: 10.21037/apm-20-1760 